\title{
Preoperative Statin Treatment Is Not Associated with Reduced Postoperative Mortality or Morbidity in Patients Undergoing Isolated Valve Surgery
}

\author{
Michel Durand, MD,${ }^{1}$ Caroline Machuron, $\mathrm{MD},{ }^{1}$ Laura Guillet, ${ }^{1} \mathrm{MD}$, Pierre-Henri Moury, MD, ${ }^{1}$ \\ Cécile Martin, MD, ${ }^{2}$ Jean-François Payen, MD, PHD,${ }^{1}$ Olivier Chavanon, MD, PHD ${ }^{2}$ \\ Pierre Albaladejo, MD, $\mathrm{PHD}^{1}$ \\ ${ }^{1}$ Pôle Anesthésie-Réanimation and 2Pôle Cardiovasculaire et Thoracique, Hopital Michallon, CHU Grenoble-Alpes, CS 21217, \\ Grenoble Cedex 9, France
}

\section{ABSTRACT}

Introduction: Previous studies have shown that statin use before coronary surgery decreases the mortality and morbidity. This benefit was not clearly detected in isolated valve surgery. The aim of this study was to assess the effect of preoperative statin therapy on postoperative complications and mortality in a large group of patients undergoing valve surgery.

Patients, Materials, and Methods: The data of consecutive patients undergoing isolated valve replacement during an 8 -year period were retrospectively reviewed from a prospective database. Mortality was compared between the patients who received preoperative statin (statin group [SG]) and those who did not receive statin (control group [CG]) after adjustment on EuroSCORE. Main postoperative complications and mortality were compared between the 2 groups by using a propensity score analysis.

Results: During the study period, 1115 patients were prospectively included, 796 in the CG group and 319 in the SG. The SG patients were significantly older, had more cardiovascular risk factors (hypertension, diabetes, and weight) than the CG patients, and benefited from more elective surgery or aortic valve replacement. No difference in mortality was found between the groups: $4.4 \%$ in the SG and $4.5 \%$ in the $\mathrm{CG}, P=.95$. Multivariate analysis also revealed no effect of statin on mortality, according to the type of surgery (aortic valve surgery alone or any kind of valve surgery) $(P=.93)$, or the elective or urgent nature of the surgery $(P=.67)$. Statin did not predict mortality after stratification with the EuroSCORE or the Parsonnet score. No difference was found between the 2 groups for postoperative complications (24-hour bleeding, atrial fibrillation, renal failure, length of mechanical ventilation, or hospital stay) and mortality after adjustment with a propensity score.

Discussion: This study found no difference in mortality or morbidity associated with preoperative statin therapy after isolated valve surgery.

Received 7uly 21, 2018; received in revised form October 6, 2018; accepted October 8, 2018.

Correspondence: Michel Durand, Pôle Anesthésie-Réanimation, Hopital Michallon, CHU Grenoble-Alpes, 38043 Grenoble Cedex 9, France; +33676767080; fax:+33-476765975 (e-mail: MDurand@chu-grenoble.fr).

\section{INTRODUCTION}

Statins reduce cholesterol and decrease cardiovascular mortality in primary and secondary prevention [Baigent 2005]. The benefit of statins during the perioperative period of coronary bypass grafting has been previously demonstrated [Pan 2004]. This benefit is attributed to the effect on cholesterol but also to pleiotropic effects, including stabilization of atheroma plaque and improvement of endothelial function [Beckman 2004]. However, results of coronary surgery studies are conflicting; some studies are positive [Pan 2004] [25], although others are negative [Ali 2005; Subramaniam 2008]. Several metaanalyses concluded that statins should be used before coronary surgery [Hindler 2006; Liakopoulos 2008]. The major complications after cardiac surgery are attributed to the inflammatory response generated by extracorporeal cardiopulmonary bypass (CPB). Thus, statins may have a beneficial effect in every cardiac procedure because of these pleiotropic effects [Martínez-Comendador 2009]. Concerning valve surgery, the benefit of a preoperative statin treatment was suggested in a recent metaanalysis [Cheng 2015], but not found in another [Kuhn 2013].

The aim of this study was to assess the effect of preoperative statin therapy on postoperative complications and mortality in a large group of patients undergoing valve surgery.

\section{PATIENTS, MATERIALS, AND METHODS}

In this monocentric retrospective study, all patients undergoing an isolated valve surgery were included during an 8-year period (January 2006 to January 2014). Patients undergoing combined valve and coronary bypass or thoracic aortic surgery were excluded. The data were collected from a prospective database with demographics, preoperative risk factors and risk scores, medical history, surgical data, and length of stay in hospital and/or ICU 30 days postoperatively. The 8-year period of analysis was divided into 3 periods that were included in the multivariate analysis (period 1: January 2006 to October 2008, $\mathrm{n}=371$; period 2: November 2008 to July 2011, $\mathrm{n}=371$; and period 3: January 2008 to January 2014, $\mathrm{n}=373$ ). Perioperative management, including anesthesia, monitoring techniques, and myocardial protection, was standardized for all patients. 
CPB was conducted by using moderate hypothermia. Surgeries were performed mainly by sternotomy. Myocardial protection was obtained by anterograde injection of cold blood cardioplegia with retrograde reinjection every 20 minutes and a warm reperfusion just before the removal of the aortic cross-clamp. Patients were classified into 2 groups: the statin group (SG) included those treated with statins preoperatively regardless of the molecule, dosage, or time of introduction before surgery. The molecules used were atorvastatin, simvastatin, rosuvastatin, and fluvastatin. The control group (CG) included patients who did not receive statin therapy preoperatively. In the SG, statins were maintained until the day before surgery and resumed on postoperative day 1 .

The primary objective was to assess the effect of preoperative statin therapy on 30-day mortality after adjustment with EuroSCORE [Nashef 1999]. The primary endpoint was postoperative mortality for all causes related to the operation and was defined as death within 30 days after surgery even if it occurred after hospital discharge.

Secondary endpoints were the association between preoperative statin therapy and severe adverse postoperative events until hospital discharge:

- Hospital mortality after adjustment with Parsonnet score [Parsonnet 1989]

- A stroke defined by the onset of a permanent deficit for more than 24 hours

- A postoperative atrial fibrillation recorded on ECG monitoring during hospitalization

- A low cardiac output syndrome as defined by a cardiac index $<2 \mathrm{~L} / \mathrm{min} / \mathrm{m}^{2}$

- The use of dobutamine during more than 3 hours at a dose $\leq 5 \mu \mathrm{gr} / \mathrm{kg}$ per min, or $\geq 5 \mu \mathrm{gr} / \mathrm{kg}$ per min

- Myocardial damage evaluated by troponin maximum value during the first 24 postoperative hours

- Acute kidney injury defined by an elevation of serum creatinine above $200 \mu \mathrm{mol} / \mathrm{L}$ or the need for renal replacement therapy

- Postoperative creatinine maximum value in intensive care

- C-reactive protein level on day 1

- Bleeding 24 hours after arrival in ICU

- Length of mechanical ventilation

- Length of stay in ICU and in the hospital

Since patients receiving statins were different from other patients, the frequency of major complications and mortality were compared between the CG and SG after matching by using a propensity score.

\section{Statistical Analysis}

Results are expressed as mean and standard deviation, and categorical data as proportion. Numbers are presented with 2 significant digits. The chi-square test was used for qualitative data, and Student $t$ test for quantitative data. A value of $\mathrm{P}<.05$ was considered significant.

Because the study was not randomized, the patients receiving preoperative stains may not have had the same risk of postoperative adverse outcomes as those who did not. To predict postoperative outcomes, models were constructed with the EuroSCORE and the Parsonnet score and preoperative statin treatment; the area under the curve (AUC) of the receiver operating characteristic (ROC) was also calculated. Model calibrations were assessed by using Hosmer-Lemeshow statistics. Then, a propensity score was developed by logistic regression including variables associated with statin intake $(P<.20)$ to assess the probability that a patient would receive statins. For each patient taking statins, a match was made with the patient not taking statins and with the closest propensity score. An AUC of the propensity score model for exposure was provided. The statistical analysis was performed with MedCalc software (MedCalc for Windows, version 12.5, MedCalc Software, Ostend, Belgium).

\section{RESULTS}

Among the 1115 patients with valve surgery included during the 8 -year period, $319(28.6 \%)$ patients were treated with statin before surgery. This percentage increased significantly from $23 \%$ in period 1 to $30.2 \%$ in period 2 and $32.8 \%$ in period $3(P<.001)$.

The characteristics of the population and intraoperative data are presented in Table 1. The SG patients were older with a higher body mass index, had more frequent diabetes or hypertension, and had higher EuroSCORE and Parsonnet scores.

\section{Mortality}

Thirty-day postoperative mortality was similar between both groups: $4.4 \%$ in the SG $(2.4 \%-7.4 \%)$ and $4.5 \%$ in the CG $(3.2 \%-6.2 \%)(P=.95)$. The multivariate analysis did not find any difference in mortality due to the type of surgery (aortic valve alone or aortic plus mitral valve, $P=.93$ ), or the degree of urgency (elective or urgent, $P=.67$ ).

To determine whether preoperative statin therapy was independently associated with a significant reduction in mortality, we used a logistic regression model to control for preoperative risk factors by using either the EuroSCORE or the Parsonnet score. The univariate discriminative power of EuroSCORE in predicting in-hospital mortality was well calibrated $(P$ values associated with Hosmer-Lemeshow statistics were 0.84), and EuroSCORE had a good discriminative power (AUC ROC: $0.83,95 \%$ CI: 0.81-0.86). Addition of the SG in the logistic regression did not increase the discrimination of EuroSCORE (AUC ROC: 0.83, 95\% CI: 0.81-0.86) or change the calibration ( $P$ values associated with HosmerLemeshow statistics were 0.88 ).

The univariate discriminative power of the Parsonnet score in predicting in-hospital mortality was also correctly calibrated $(P$ values associated with Hosmer-Lemeshow statistics were 0.74 ), with a good discriminative power (AUC ROC: $0.80,95 \%$ CI: 0.78-0.82). Inclusion of the SG did not increase the discrimination of the Parsonnet score (AUC ROC: $0.80,95 \%$ CI: 0.78-0.82) but decreased the calibration $(P$ values associated with Hosmer-Lemeshow statistics were 0.06$)$. 
Table 1. Demographic, Clinical, and Intraoperative Characteristics*

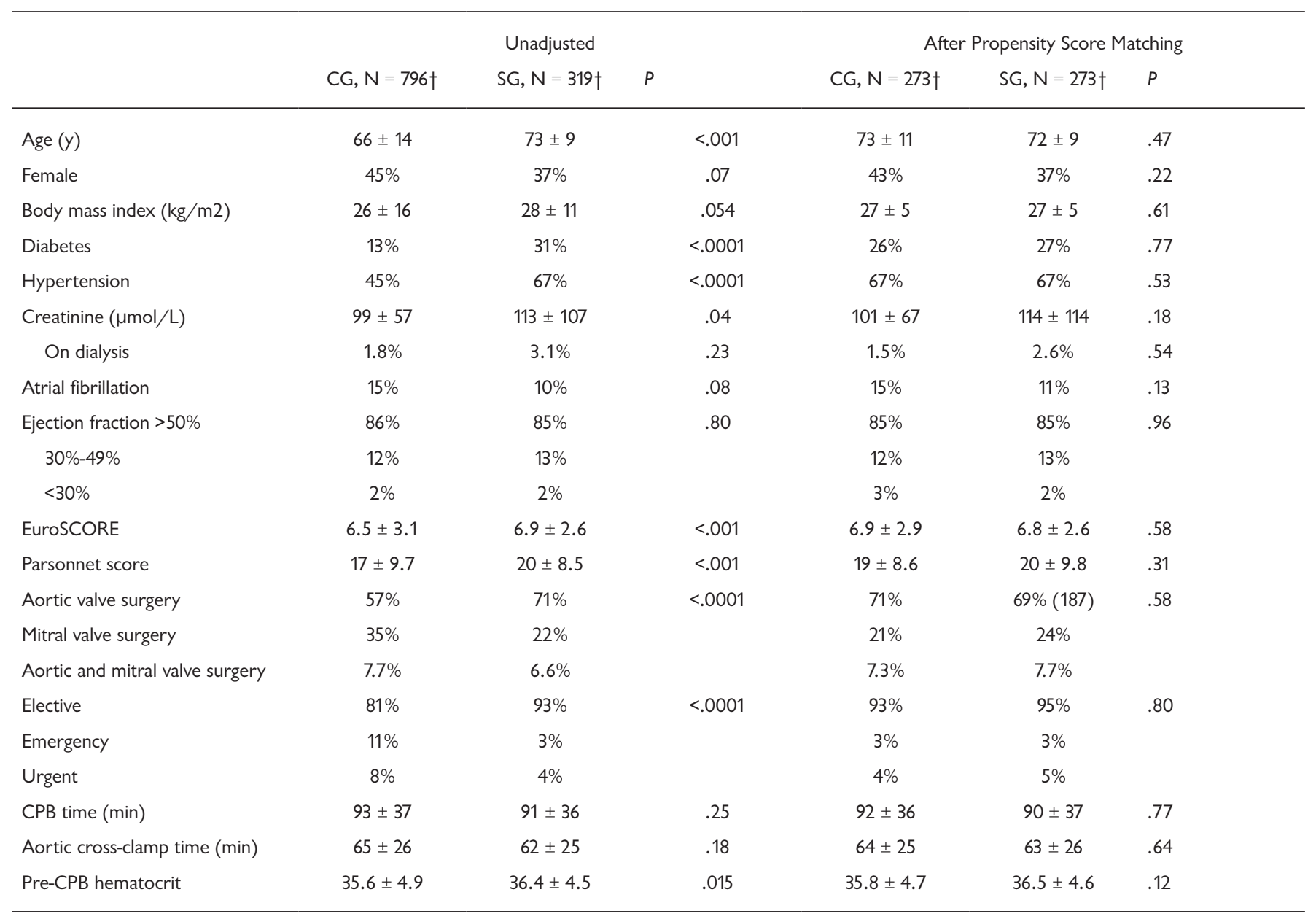

*CG, control group; SG, statin group.

$\dagger$ Data expressed as mean \pm standard deviation or as percentage.

\section{Morbidity}

No significant difference in postoperative complications was found between the 2 groups, including postoperative use of dobutamine, renal failure, atrial fibrillation, stroke, length of ventilation, length of stay in ICU, or length of stay in the hospital (Table 2).

\section{After Propensity Score Adjustment}

Two comparable groups of 273 patients were obtained after propensity score matching of variables associated with statin therapy: age, sex, EuroSCORE, hypertension, preoperative diabetes, body mass index, surgery period, type of surgery (mitral, aortic, or double valve), and degree of urgency. AUC of the propensity score model for exposure was 0.73 , and the Hosmer-Lemeshow test was negative $(P=.71)$. After controlling for the propensity score, we found no difference in the main postoperative complications or mortality in the statin-treated group (Table 2).

\section{DISCUSSION}

This study found that preoperative statin treatment was not associated with a reduction in 30-day mortality after isolated valve surgery. Similarly, no benefit was found for postoperative complications studied, atrial fibrillation, renal failure, or low cardiac output, regardless of the statistical method used.

The main complications after cardiac surgery are cardiovascular (infarction, low cardiac output syndrome, and/ or arrhythmia), surgical (bleeding), and respiratory, renal, or neurological. The mechanisms of these complications include inflammatory phenomena caused by CPB (cytokines, interleukins, etc.), thrombosis, and cardiovascular events that could potentially be reduced by statin. A beneficial effect of statins was reported after coronary surgery on several complications, including major adverse cardiac events [Thielmann 2007], stroke [Berwanger 2016], delirium [Katznelson 2009], and renal failure [Layton 2013]. Hence, 
Table 2. Postoperative Complications in the Whole Population and after Adjustment with a Propensity Score*

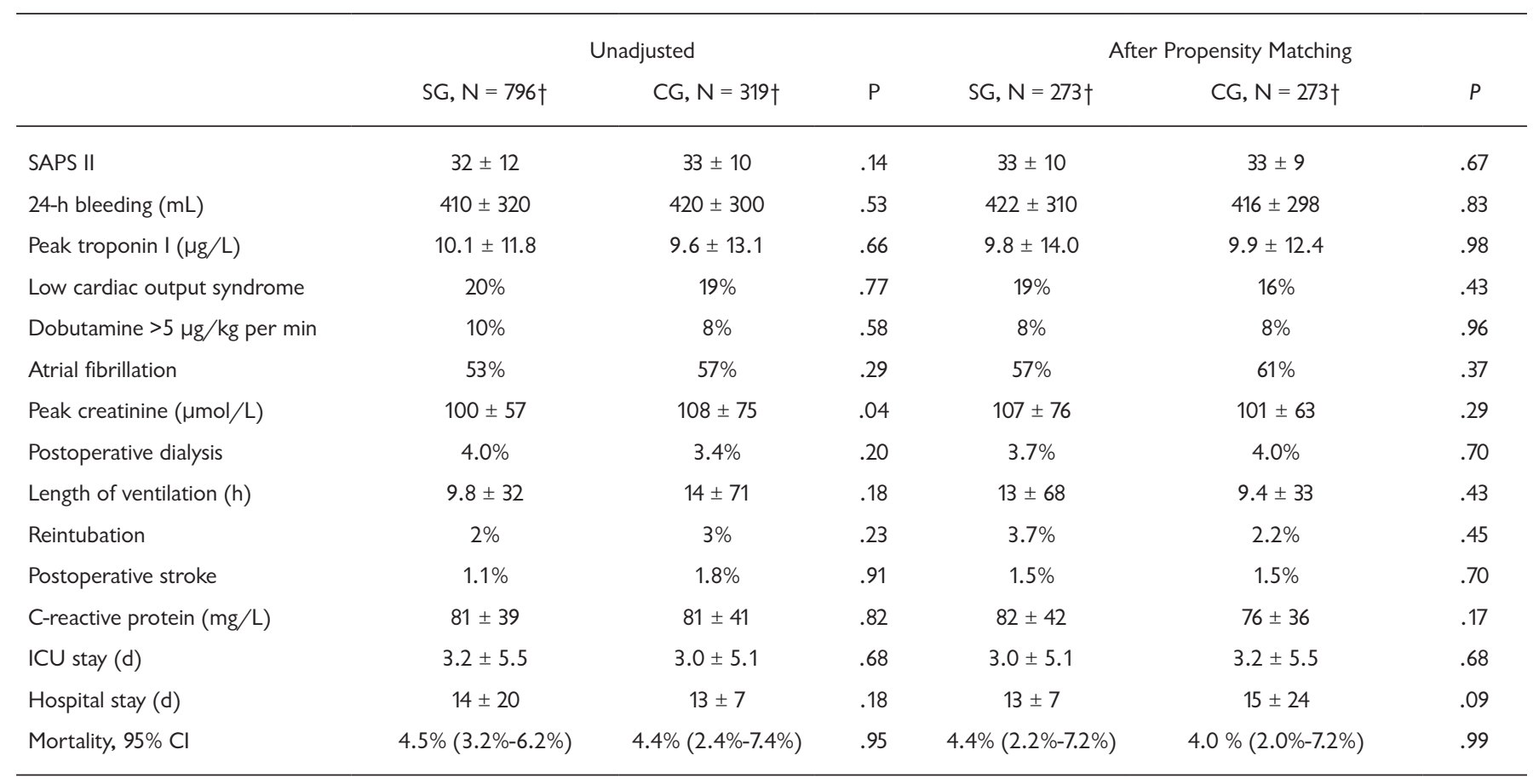

*CG, control group; SG, statin group.

†Data expressed as mean \pm standard deviation, as percentage, or as percentage $(95 \% \mathrm{CI})$.

the same beneficial effects should potentially be expected in other cardiac surgeries.

A metaanalysis [Liakopoulos 2008] including 19 studies and more than 30,000 patients found a $43 \%$ reduction in mortality associated with the preoperative administration of a statin. This metaanalysis included patients following valve, coronary, or combined cardiac surgeries. Regarding isolated valve surgery, several studies have found positive results. A study of 3217 patients in 2 different centers found a decrease in mortality associated with preoperative statin intake and a decrease in complications [Angeloni 2011]. Another study including 10,061 patients who had both valve or combined surgery did not find any change in mortality associated with statin therapy [Borger 2010].

A randomized study demonstrated a significant reduction in the rate of postoperative atrial fibrillation after cardiac surgery in patients treated with statin [Patti 2006]. Statins did not reduce the incidence of atrial fibrillation after isolated valve surgery [Folkeringa 2011]. The 4 studies on valve surgery included in a metaanalysis did not report atrial fibrillation data [Fedoruk 2008]. In our study, statins were not associated with a lower incidence of atrial fibrillation in the whole population or after propensity score matching. No reduction in the frequency of postoperative myocardial infarction was found in a metaanalysis [Liakopoulos 2008].

We found no beneficial effect of statin therapy on renal function. A beneficial effect on renal function associated with statin use was reported in one study [Layton 2013]. However, a study of 447 patients undergoing isolated valve surgery found no decrease in postoperative renal failure [Fedoruk 2008]. One metaanalysis failed to demonstrate a positive effect of statins on renal function [Guay 2014], and a randomized prospective study using an accurate classification of renal insufficiency did not find any significant improvement [Prowle 2012].

We found no beneficial effect on the incidence of stroke. Our study was underpowered for this complication. However, similar results are found in an observational study [Koenig 2009] and in a metaanalysis [Cheng 2015].

The benefits of statins are mainly attributed to a decrease in proinflammatory cytokine production produced by $\mathrm{CPB}$ [Chello 2007] and the preservation of endothelial function [Chello 2005]. The effects of statins seem to be independent of the patient's lipid status [Mannacio 2008] and are more pronounced in patients with preoperative activation of the inflammatory system [Martínez-Comendador 2013]. The impact of cardiovascular risk factors emerges with a beneficial effect of statins in isolated valve surgery found mainly in patients who already have cardiovascular risk factors [Allou 2010]. Thus, the effect seems related to a patient's cardiovascular profile. Many studies are observational or retrospective, and differences in selected populations may explain the wide variability of the reported results.

This study also had limitations: it was a retrospective study and it was not known for each patient the type, dosage, or duration of statin intake before surgery. The long collection period can be a source variation in population and perioperative management. As a propensity analysis considers only 
known factors, there might be unmeasured factors associated with statin treatment that have influenced morbidity and mortality. Another limitation was that different statins were included: a recent study shows that the different statins are nearly equivalent [Naci 2013]. The last limitation is the power of the study: mortality was a rare event: the observed mortality was $4.5 \%$; the study could detect a $3 \%$ decrease in mortality in the SG. However, the power of the study was better for morbidity whose frequency was higher: the study could detect an $8 \%$ decrease in the atrial fibrillation rate.

\section{CONCLUSION}

Preoperative statin therapy before valve surgery did not reduce the risks of mortality or complications. Statins can be maintained before and after surgery, but the results of this study do not support the preoperative initiation of statin therapy before valve surgery in order to reduce complications or mortality.

\section{REFERENCES}

Ali IS, Buth KJ. 2005. Preoperative statin use and outcomes following cardiac surgery. Int J Cardiol 103:12-8.

Allou N, Augustin P, Dufour G, et al. 2010. Preoperative statin treatment is associated with reduced postoperative mortality after isolated cardiac valve surgery in high-risk patients. J Cardiothorac Vasc Anesth 24: 921-6.

Angeloni E, Melina G, Benedetto U, et al. 2011. Statins improve outcome in isolated heart valve operations: a propensity score analysis of 3,217 patients. Ann Thorac Surg 92:68-73.

Baigent C, Keech A, Kearney PM, et al. 2005. Efficacy and safety of cholesterol-lowering treatment: prospective meta-analysis of data from 90056 participants in 14 randomised trials of statins. Lancet 366:1267-78. Group author name: Cholesterol Treatment Trialists' (CTT) Collaborators.

Beckman JA, Liao JK, Hurley S, et al. 2004. Atorvastatin restores endothelial function in normocholesterolemic smokers independent of changes in low-density lipoprotein. Circ Res 95:217-23.

Berwanger O, Le Manach Y, Suzumura EA, et al. 2016. Association between pre-operative statin use and major cardiovascular complications among patients undergoing non-cardiac surgery: the VISION study. Eur Heart J 37:177-85.

Borger MA, Seeburger J, Walther T, et al. 2010. Effect of preoperative statin therapy on patients undergoing isolated and combined valvular heart surgery. Ann Thorac Surg 89:773-9

Chello M, Anselmi A, Spadaccio C, et al. 2007. Simvastatin increases neutrophil apoptosis and reduces inflammatory reaction after coronary surgery. Ann Thorac Surg 83:1374-80.

Chello M, Goffredo C, Patti G, et al. 2005. Effects of atorvastatin on arterial endothelial function in coronary bypass surgery. Eur J Cardiothorac Surg 28:805-10.

Cheng X, Hu Q, Liu Z, Tang X. 2015. Preoperative statin therapy decreases early mortality in patients undergoing isolated valve surgery: result from a meta-analysis. J Cardiothorac Vasc Anesth 29:107-14.

Fedoruk LM, Wang H, Conaway MR, Kron IL, Johnston KC. 2008.
Statin therapy improves outcomes after valvular heart surgery. Ann Thorac Surg 85: 1521-5; discussion 1525-6.

Folkeringa RJ, Tieleman RG, Maessen JG, Prins MH, Nieuwlaat R, Crijns HJ. 2011. Statins do not reduce atrial fibrillation after cardiac valvular surgery: a single centre observational study. Neth Heart J 19:17-23.

Guay J, Ochroch EA. 2014. Effects of adding statins before surgery on mortality and major morbidity: a meta-analysis. J Cardiothorac Vasc Anesth 28:255-66.

Hindler K, Shaw AD, Samuels J, Fulton S, Collard CD, Riedel B. 2006. Improved postoperative outcomes associated with preoperative statin therapy. Anesthesiology 105:1260-72; quiz 1289-90.

Katznelson R, Djaiani GN, Borger MA, et al. 2009. Preoperative use of statins is associated with reduced early delirium rates after cardiac surgery. Anesthesiology 110: 67-73.

Koenig MA, Grega MA, Bailey MM, et al. 2009. Statin use and neurologic morbidity after coronary artery bypass grafting: a cohort study. Neurology 73:2099-106.

Kuhn EW, Liakopoulos OJ, Stange S, et al. 2013. Meta-analysis of patients taking statins before revascularization and aortic valve surgery. Ann Thorac Surg 96:1508-16.

Layton JB, Kshirsagar AV, Simpson RJ Jr, et al. 2013. Effect of statin use on acute kidney injury risk following coronary artery bypass grafting. Am J Cardiol 111:823-8.

Liakopoulos OJ, Choi YH, Haldenwang PL, et al. 2008. Impact of preoperative statin therapy on adverse postoperative outcomes in patients undergoing cardiac surgery: a meta-analysis of over 30,000 patients. Eur Heart J 29:1548-59.

Mannacio VA, Iorio D, De Amicis V, Di Lello F, Musumeci F. 2008. Effect of rosuvastatin pretreatment on myocardial damage after coronary surgery: a randomized trial. J Thorac Cardiovasc Surg 136:1541-8.

Martínez-Comendador J, Alvarez JR, Mosquera I, et al. 2009. Preoperative statin treatment reduces systemic inflammatory response and myocardial damage in cardiac surgery. Eur J Cardiothorac Surg 36:998-1005.

Martínez-Comendador J, Alvarez JR, Sierra J, Teijeira E, Adrio B. 2013. Preoperative statin therapy in cardiac surgery is more effective in patients who display preoperative activation of the inflammatory system. Tex Heart Inst J 40:42-9.

Naci H, Brugts JJ, Fleurence R, Ades AE. 2013. Dose-comparative effects of different statins on serum lipid levels: a network meta-analysis of 256,827 individuals in 181 randomized controlled trials. Eur J Prev Cardiol 20:658-70.

Nashef SA, Roques F, Michel P, Gauducheau E, Lemeshow S, Salamon R. 1999. European system for cardiac operative risk evaluation (EuroSCORE). Eur J Cardiothorac Surg 16:9-13.

Pan W, Pintar T, Anton J, Lee VV, Vaughn WK, Collard CD. 2004. Statins are associated with a reduced incidence of perioperative mortality after coronary artery bypass graft surgery. Circulation 110(11 suppl 1):II45-9.

Parsonnet V, Dean D, Bernstein AD. 1989. A method of uniform stratification of risk for evaluating the results of surgery in acquired adult heart disease. Circulation 79(6 Pt 2):I3-12.

Patti G, Chello M, Candura D, et al. 2006. Randomized trial of atorvastatin for reduction of postoperative atrial fibrillation in patients undergoing cardiac surgery: results of the ARMYDA-3 (Atorvastatin for Reduction of MYocardial Dysrhythmia After cardiac surgery) study. Circulation 114:1455-61. 
Prowle JR, Calzavacca P, Licari E, et al. 2012. Pilot double-blind, randomized controlled trial of short-term atorvastatin for prevention of acute kidney injury after cardiac surgery. Nephrology (Carlton) $17: 215-24$.

Subramaniam K, Koch CG, Bashour A, et al. 2008. Preoperative statin intake and morbid events after isolated coronary artery bypass grafting. J Clin Anesth 20:4-11.

Thielmann M, Neuhäuser M, Marr A, et al. 2007. Lipid-lowering effect of preoperative statin therapy on postoperative major adverse cardiac events after coronary artery bypass surgery. J Thorac Cardiovasc Surg 134:1143-9. 